\title{
ALMA MEASUREMENTS OF CIRCUMSTELLAR MATERIAL IN THE GQ LUP SYSTEM
}

\author{
Meredith A. MacGregor ${ }^{1}$, David J. Wilner ${ }^{1}$, Ian Czekala ${ }^{1,2}$, Sean M. Andrews ${ }^{1}$, Y. Sophia Dai ${ }^{3}$, \\ Gregory J. Herczeg ${ }^{4}$, Kaitlin M. Kratter ${ }^{5}$, Adam L. Kraus ${ }^{6}$, Luca Ricci ${ }^{1}$, And Leonardo Testi ${ }^{7}$ \\ ${ }^{1}$ Harvard-Smithsonian Center for Astrophysics, 60 Garden Street, Cambridge, MA 02138, USA \\ ${ }^{2}$ Kavli Institute for Particle Astrophysics and Cosmology (KIPAC), Stanford University, Stanford, CA 94305, USA \\ ${ }^{3}$ Caltech/IPAC, 1200 E. California Boulevard, Pasadena, CA, 91125, USA \\ ${ }^{4}$ Kavli Institute for Astronomy and Astrophysics, Peking University, Yi He Yuan Lu 5, Haidian Qu, 100871 Beijing, China \\ ${ }^{5}$ Department of Astronomy, University of Arizona, Tucson, AZ 85721, USA \\ ${ }^{6}$ Department of Astronomy, The University of Texas at Austin, Austin, TX 78712, USA \\ ${ }^{7}$ European Southern Observatory (ESO) Headquarters, Karl-Schwarzschild-Str. 2, D-85748 Garching bei Muenchen, Germany \\ Received 2016 September 23; revised 2016 November 11; accepted 2016 November 17; published 2017 January 16
}

\begin{abstract}
We present Atacama Large Millimeter/submillimeter Array observations of the GQ Lup system, a young Sun-like star with a substellar-mass companion in a wide-separation orbit. These observations of $870 \mu \mathrm{m}$ continuum and CO $J=3-2$ line emission with beam size $\sim 0$ " $3(\sim 45 \mathrm{au})$ resolve the disk of dust and gas surrounding the primary star, GQ Lup A, and provide deep limits on any circumplanetary disk surrounding the companion, GQ Lup b. The circumprimary dust disk is compact with an FWHM of $59 \pm 12 \mathrm{au}$, while the gas has a larger extent with a characteristic radius of $46.5 \pm 1.8 \mathrm{au}$. By forward-modeling the velocity field of the circumprimary disk based on the $\mathrm{CO}$ emission, we constrain the mass of GQ Lup A to be $M_{*}=(1.03 \pm 0.05) *(d / 156 \mathrm{pc}) M_{\odot}$, where $d$ is a known distance, and determine that we view the disk at an inclination angle of $60^{\circ} .5 \pm 0.5$ and a position angle of $346^{\circ} \pm 1^{\circ}$. The $3 \sigma$ upper limit on the $870 \mu \mathrm{m}$ flux density of any circumplanetary disk associated with GQ Lup b of $<0.15 \mathrm{mJy}$ implies an upper limit on the dust disk mass of $<0.04 M_{\oplus}$ for standard assumptions about optically thin emission. We discuss proposed mechanisms for the formation of wide-separation substellar companions given the non-detection of circumplanetary disks around GQ Lup b and other similar systems.
\end{abstract}

Key words: circumstellar matter - stars: individual (GQ Lup) - submillimeter: planetary systems

\section{INTRODUCTION}

Direct-imaging surveys for extrasolar planets are revealing a surprising population of low-mass companions at wideseparations (semimajor axis $>100$ au; Chauvin et al. 2005; Luhman et al. 2006; Lafrenière et al. 2008; Ireland et al. 2011; Kraus et al. 2014; Bowler et al. 2015; Kraus et al. 2015). These substellar $\left(<40 M_{\text {Jup }}\right)$ companions present serious challenges to standard models of both planet and binary star formation (e.g., Debes \& Sigurdsson 2006). Conventional "core accretion" models struggle to form such massive objects at large semimajor axes (Pollack et al. 1996; Lambrechts \& Johansen 2012), while core fragmentation and gravitational instability are difficult to arrest at low masses and preferentially form more massive objects (Bate et al. 2003; Jiang et al. 2004; Boley et al. 2010; Kratter et al. 2010). Another possibility is that these objects formed closer in to their host stars and were subsequently scattered (or migrated) outwards through dynamical interactions with another close in companion (Boss 2006; Crida et al. 2009).

The growing population of wide-separation companions offers a new window to explore the processes of giant planet assembly and the subsequent formation of moon systems. Several of these companions exhibit line emission, as well as infrared and ultraviolet excesses commonly associated with ongoing accretion from "circumplanetary" disks (Seifahrt et al. 2007; Schmidt et al. 2008; Bowler et al. 2011, 2014; Bailey et al. 2013; Zhou et al. 2014). There is also evidence for circumplanetary disks around planets at closer separations from photometric transit surveys (J1407, see Mamajek et al. 2012). Models of giant planet formation make testable predictions about the size, scale height, and mass distribution of these circumplanetary disks (e.g., Ayliffe \& Bate 2009). Furthermore, the properties of these disks govern the composition and orbits of any moons that may form (Heller et al. 2014).

One of the most prominent and best characterized examples of a system with a directly imaged low-mass, wide-separation companion with evidence for a circumplanetary disk is GQ Lup. We present new observations of $870 \mu \mathrm{m}$ continuum and CO $J=3-2$ line emission from the GQ Lup system made with the Atacama Large Millimeter/submillimeter Array (ALMA). These new ALMA observations place a stringent upper limit on the emission from any circumplanetary disk surrounding GQ Lup b, and they provide strong constraints on the geometry of the disk surrounding GQ Lup A. We introduce the GQ Lup system in Section 2. In Section 3, we present the ALMA observations. In Section 4, we describe the analysis techniques and results for both continuum and line emission. In Section 5, we discuss the significance of the results on the circumprimary disk geometry, the limit on a circumplanetary disk, and implications for the formation mechanisms of wide-separation, substellar companions.

\section{THE GQ LUP SYSTEM}

The GQ Lup system is located in the $3 \pm 2$ Myr old (Alcalá et al. 2014) Lupus I cloud (Tachihara et al. 1996) at a distance of $156 \pm 50 \mathrm{pc}$ (determined from parallax, see Neuhäuser et al. 2008). New parallax measurements from Gaia DR1 for stars in Lupus I, yield an average parallax of $6.4 \pm 0.3$ mas or $156.3 \pm 7.3 \mathrm{pc}$ (Lindegren et al. 2016), comparable to the earlier parallax measurements. The primary star, GQ Lup A, is a classical T Tauri star (spectral type K7V, Kharchenko \& Roeser 2009), with a photospheric temperature of 4000-4300 K (Donati et al. 2012; Pecaut \& Mamajek 2013; Herczeg \& Hillenbrand 2014). Seperuelo Duarte et al. (2008) estimate a 
Table 1

ALMA Observations of GQ Lup

\begin{tabular}{lcccc}
\hline \hline $\begin{array}{l}\text { Observation } \\
\text { Date }\end{array}$ & $\begin{array}{c}\text { \# of } \\
\text { Antennas }\end{array}$ & $\begin{array}{c}\text { Projected } \\
\text { Baselines }(\mathrm{m})\end{array}$ & $\begin{array}{c}\text { PWV } \\
(\mathrm{mm})\end{array}$ & $\begin{array}{c}\text { Time on } \\
\text { Target (minutes) }\end{array}$ \\
\hline 2015 Jun 14 & 41 & $16-784$ & 0.6 & 31.3 \\
2015 Jun 15 & 37 & $21-784$ & 0.4 & 31.1 \\
2015 Aug 28 & 40 & $15-1574$ & 1.1 & 35.0 \\
\hline
\end{tabular}

stellar radius of $1.8 \pm 0.3 R_{\odot}$ and assume an effective temperature of $4060 \mathrm{~K}$ to determine a stellar luminosity of $0.8 \pm 0.3 L_{\odot}$. Although they adopt a much higher effective temperature of $4300 \pm 50 \mathrm{~K}$, Donati et al. (2012) obtain a comparable stellar radius of $1.7 \pm 0.2 R_{\odot}$. Previous estimates of the mass of GQ Lup A vary between 0.7 and $1.05 M_{\odot}$, largely depending on the evolutionary models and effective temperatures used (Mugrauer \& Neuhäuser 2005; Seperuelo Duarte et al. 2008; Donati et al. 2012). Adopting the higher effective temperature of $4300 \mathrm{~K}$ yields a mass of $1.05 \pm$ $0.07 M_{\odot}$, the upper value in this range (Donati et al. 2012). Additionally, GQ Lup A possesses strong mid- and far-infrared excesses, indicative of a circumstellar disk (Hughes et al. 1994). Dai et al. (2010) marginally resolved $1.3 \mathrm{~mm}$ dust emission from the circumstellar disk using the Submillimeter Array (SMA) and determined an outer radius of $<75 \mathrm{au}$.

The substellar companion, GQ Lup b, was discovered by Neuhäuser et al. (2005) using the Hubble Space Telescope (HST). By fitting to the broadband spectral energy distribution, Zhou et al. (2014) determine that the companion has a radius of $6.5 \pm 2 R_{\mathrm{Jup}}$, an effective temperature of $2050 \pm 350 \mathrm{~K}$, and a luminosity of $\log L_{\text {phot }} / L_{\odot}=-2.25 \pm 0.24$. The mass of this companion is uncertain, with estimates ranging from 10-36 $M_{\text {Jup }}$ (Marois et al. 2007; Seifahrt et al. 2007; Neuhäuser et al. 2008; Lavigne et al. 2009). The projected separation of the companion from the primary star is 0.7 (Ginski et al. 2014), and recent work by Schwarz et al. (2016) favors orbits with high eccentricity and semimajor axes 100-185 au. Near-infrared spectroscopy by Seifahrt et al. (2007) showed $\mathrm{Pa} \beta$ line emission (equivalent width, $\mathrm{EW}=-3.83 \pm 0.12 \AA$ ), though subsequent observations by Lavigne et al. (2009) give a limit an order of magnitude lower for the same line (EW $=-0.46 \pm 0.08 \AA$ ), possibly pointing to time variability of $\mathrm{Pa} \beta$ and ongoing disk accretion. Optical photometery using $H S T$ shows a significant blue excess that corresponds to an accretion rate $\sim 5 \times 10^{-10} M_{\odot} \mathrm{yr}^{-1}$ (Zhou et al. 2014).

\section{OBSERVATIONS}

The GQ Lup system was observed with ALMA in Band 7 $(870 \mu \mathrm{m})$ in a one-hour (total of $\sim 30$ minutes on-source) Scheduling Block (SB) on 2015 June 14 with 41 operational antennas and on 2015 June 15 with 37 operational antennas, using baselines that spanned 15 to $784 \mathrm{~m}$. An additional onehour SB was executed on 2015 August 28 with 40 operational antennas, using baselines reaching to $1574 \mathrm{~m}$. These observations are summarized in Table 1, including the dates, baseline lengths, weather conditions, and time on-source. Overall, the weather was very good (pwv $\lesssim 1.1 \mathrm{~mm}$ ). The correlator was configured to optimize continuum sensitivity, while including both the ${ }^{12} \mathrm{CO}$ and ${ }^{13} \mathrm{CO} \mathrm{J}=3-2$ transitions at 345.79599 and $330.58797 \mathrm{GHz}$, respectively. The setup used four basebands, centered at $331,333,343$, and $345 \mathrm{GHz}$, in two polarizations. The basebands with the targeted spectral lines, centered at 331 and $345 \mathrm{GHz}$, each have 3840 channels over a bandwidth of $1.875 \mathrm{GHz}$, while the other two basebands each have only 128 channels over a bandwidth of $2 \mathrm{GHz}$. The phase center for the June observations was specified at $\alpha=15^{\mathrm{h}} 49^{\mathrm{m}} 12.082861$, $\delta=-35^{\circ} 39^{\prime} 05^{\prime \prime} 48071$ (J2000), and the phase center for the August observations was $\alpha=15^{\mathrm{h}} 49^{\mathrm{m}} 12.082607, \delta=-35^{\circ} 39^{\prime}$ 05!"48550. These phase centers correspond to the J2000 position of the star corrected for its proper motion of $(-15.1$, -23.4) $\mathrm{mas} \mathrm{yr}^{-1}$. The field of view at the center frequency of $338 \mathrm{GHz}$ is $\sim 18^{\prime \prime}$, set by the FWHM primary beam size of the $12 \mathrm{~m}$ diameter array antennas.

The raw data sets were delivered with calibration scripts provided by ALMA staff. We executed these scripts for each SB using the CASA package (version 4.5.0) to generate calibrated visibilities. Time-dependent gain variations due to atmospheric and instrumental effects were corrected using interleaved observations of the calibrator J1534-3526. Bandpass calibration was determined from observations of J15172422. The absolute flux calibration was derived from observations of Titan and Ceres, with a systematic uncertainty estimated at less than $10 \%$. A single iteration of phase-only self-calibration was employed, after which the visibilities were averaged into $30 \mathrm{~s}$ intervals. We generated both continuum and $\mathrm{CO}$ line images using the multi-frequency synthesis CLEAN algorithm in CASA. For spectral line imaging, the continuum level was subtracted from the spectral windows containing emission lines.

\section{RESULTS AND ANALYSIS}

\subsection{Continuum Emission}

Figure 1 (left panel) shows the ALMA $870 \mu \mathrm{m}$ continuum emission. With robust $=0.5$ weighting, the synthesized beam size is 0 ". $37 \times 0$ "! $23(58 \times 36$ au at $156 \mathrm{pc})$ with a position angle of $-87^{\circ}$, and the rms noise level is $50 \mu \mathrm{Jy} /$ beam. This image reveals compact dust continuum emission around GQ Lup A (star symbol) and no evidence for emission at the position of GQ Lup b (diamond symbol). From this nondetection, we determine a $3 \sigma$ upper limit on the flux density of any circumplanetary disk surrounding the secondary of $<0.15 \mathrm{mJy}$ (assuming a point source). The right panel of Figure 1 shows the deprojected real visibilities averaged in bins of $u, v$ distance, centered on GQ Lup A using the disk inclination and orientation determined by forward-modeling the $\mathrm{CO}$ emission (see Section 4.2 for a detailed description). The profile shows a central peak and fall-off, but without the subsequent ringing that would be expected for a simple disk with a single radial power-law surface brightness profile. A more complicated surface brightness profile (e.g., a broken power-law, see Hogerheijde et al. 2016) is more consistent, but a proper radiative transfer calculation will be needed to determine the precise radial profiles of the disk surface density and temperature. The deprojected imaginary visibilities are consistent with zero, as is expected for a symmetric structure.

By fitting a simple two-dimensional Gaussian to the continuum image, we obtain a total flux density for the circumprimary disk of $77.8 \pm 0.2 \mathrm{mJy}$, consistent with previous interferometric and single dish millimeter flux measurements. Dai et al. (2010) measure a flux density of $25 \pm 3 \mathrm{mJy}$ at $1.3 \mathrm{~mm}$ with the SMA. Nuernberger et al. (1997) measure a flux density of $38 \pm 7 \mathrm{mJy}$ at $1.25 \mathrm{~mm}$ with the SEST bolometer. If we extrapolate our ALMA 

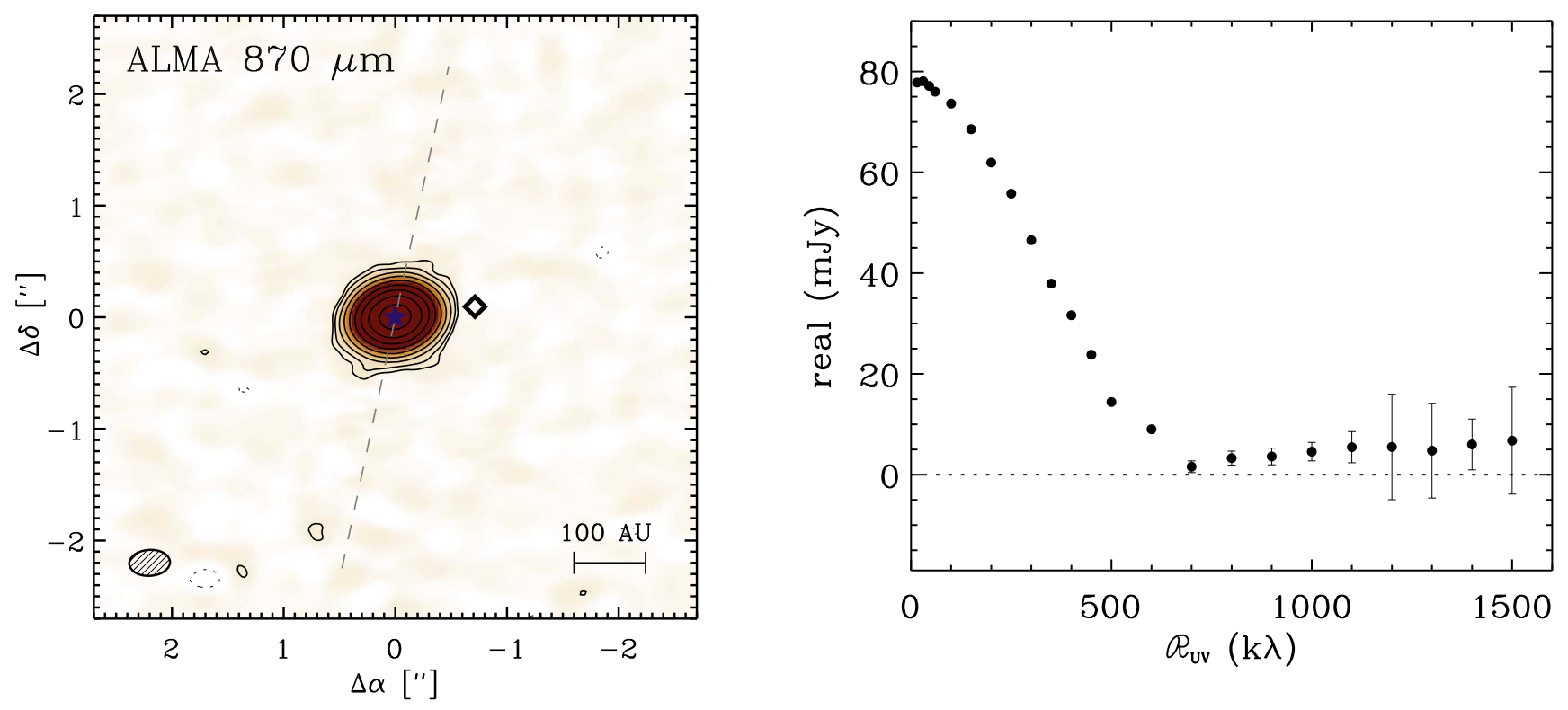

Figure 1. (Left) ALMA image of the $870 \mu \mathrm{m}$ continuum emission from GQ Lup. The contour levels are in steps of $[3,6,12,24,48, \ldots] \times 50 \mu \mathrm{Jy}$, the rms noise level in the image. The star symbol marks the position of the primary star, GQ Lup A, and the diamond indicates the projected location of the secondary companion, GQ Lup b. The dashed gray line shows the position angle of the disk major axis determined by forward-modeling the $\mathrm{CO}$ emission and the dashed ellipse indicates the 0 ." $37 \times 0$ ! 23 (FWHM) synthesized beam size. (Right) The deprojected real visibilities averaged in bins of $u, v$ distance.

measurement using a typical spectral index for $\mathrm{T}$ Tauri stars of $2.4 \pm 0.5$ (Andrews et al. 2013), we obtain a flux density at $1.3 \mathrm{~mm}$ of $29.7 \pm 5.4 \mathrm{mJy}$, in good agreement with both previous flux density measurements within their uncertainties. Given this flux density, the GQ Lup circumstellar disk is brighter than $\sim 70 \%$ of other Lupus sources with spectral types K4-M1 (Ansdell et al. 2016). The major axis FWHM of the continuum emission (deconvolved from the beam) is $0 . " 38 \pm 0$ ! 07 . At a distance of $156 \mathrm{pc}$, this gives a characteristic size for the primary disk of $59 \pm 12 \mathrm{au}$, again comparable to the results of Dai et al. (2010), who derived an outer radius for the disk of $25-50$ au ( $~ 50-100$ au in diameter). Longer baseline observations with higher angular resolution are needed to better constrain the location and sharpness of the dust disk edges, and to probe for any substructure that might betray the presence of an additional inner companion in the system.

For optically thin emission, we can make a simple estimate of the total dust mass $\left(M_{\text {dust }}\right)$ for the circumprimary disk given the observed total flux density (Hildebrand 1983):

$$
M_{\mathrm{dust}}=\frac{F_{\nu} D^{2}}{\kappa_{\nu} B_{\nu}\left(T_{\mathrm{dust}}\right)} .
$$

Here, $B_{\nu}\left(T_{\text {dust }}\right)$ is the Planck function at the dust temperature, $T_{\text {dust }}$, and $\kappa_{\nu}$ is the dust opacity. For consistency with Bowler et al. (2015) and Ansdell et al. (2016), we adopt the frequencydependent dust opacity $\kappa_{\nu}=10\left(\nu / 10^{12} \mathrm{~Hz}\right) \mathrm{cm}^{2} \mathrm{~g}^{-1}$ from Beckwith et al. (1990). At $870 \mu \mathrm{m}$, the dust opacity is $\kappa_{\nu}=3.4 \mathrm{~cm}^{2} \mathrm{~g}^{-1}$. To estimate the dust temperature, we use the dust temperature-stellar luminosity relationship of Andrews et al. (2013): $T_{\text {dust }}=25\left(L / L_{\odot}\right)^{1 / 4}$. For GQ Lup A, this relation yields $T_{\text {dust }}=24 \pm 8 \mathrm{~K}$. The resulting dust mass is $15.10 \pm 0.04 M_{\oplus}$.

Similarly, we can use the $3 \sigma$ upper limit on the flux density of a circumplanetary disk around the companion GQ Lup b to place an upper limit on the potential dust mass. Given its low luminosity, we assume that heating of a circumplanetary disk around GQ Lup b is dominated by the primary star, GQ Lup A, rather than by the companion itself. If we assume that the orbit of GQ Lup b and the circumprimary disk are coplanar, the radiative equilibrium temperature at the position of GQ Lup b ( $\sim 220 \mathrm{au}$, see Section 5.1 for discussion), is $18 \pm 2 \mathrm{~K}$. Taking this value as a representative dust temperature for our analysis, the resulting $3 \sigma$ upper limit on the dust mass is $M_{\text {dust }}<0.04 M_{\oplus}$. For a gas-to-dust ratio of 100 , this implies a total circumplanetary disk mass of $M_{\text {tot }}<4 M_{\oplus}$ or $<0.04 \%-$ $0.13 \%$ the mass of the companion itself (for companion masses of 10-36 $\left.M_{\mathrm{Jup}}\right)$. This estimate of the disk dust mass is sensitive to both the assumed dust opacity, $\kappa_{\nu}$, and the characteristic dust temperature, $T_{\text {dust }}$. Van der Plas et al. (2016) derive a temperature-luminosity relationship for spectral types M5 and later (assuming different prescriptions for disk flaring and opacity than Andrews et al. 2013): $T_{\text {dust }}=22\left(L / L_{\odot}\right)^{0.16}$. Given a luminosity of $\sim 0.006 L_{\odot}$ for GQ Lup b, this relationship implies a dust temperature of $\sim 10 \mathrm{~K}$. If we take $T_{\text {dust }}=10 \mathrm{~K}, \quad$ instead, then $M_{\text {dust }}<0.14 \quad M_{\oplus} \quad$ and $M_{\text {tot }}<14 M_{\oplus}$. Even for this low temperature the total disk mass is $\lesssim 0.1 \%-0.3 \%$ of the companion mass. Any possible viscous heating of the disk (e.g., Isella et al. 2014) is neglected here because of the low measured accretion rate, $\sim 5 \times 10^{-10} M_{\odot} \mathrm{yr}^{-1} \sim 0.5 M_{\mathrm{Jup}} \mathrm{Myr}^{-1}$. If we take the measured accretion rate together with a disk mass of $\lesssim 4-14 M_{\oplus}$ for the circumplanetary disk, this yields an expected lifetime of $\sim 2 \times 10^{4}-1 \times 10^{5} \mathrm{yr}$, shorter than the age of the GQ Lup system of $\sim 2$ Myr. The total dust mass of the circumplanetary disk also places constraints on the possibility of moon formation around the companion. In our own solar system, the total mass of the moons of Jupiter, Saturn, and Uranus are all $\sim 10^{-4}$ the mass of their host planet (Canup \& Ward 2006). The dust content of the GQ Lup b disk is at least a factor of six lower than this moon-planet mass ratio, making it difficult to form gas giant moons. However, in the model of Canup \& Ward (2006) satellites form in a circumplanetary disk 

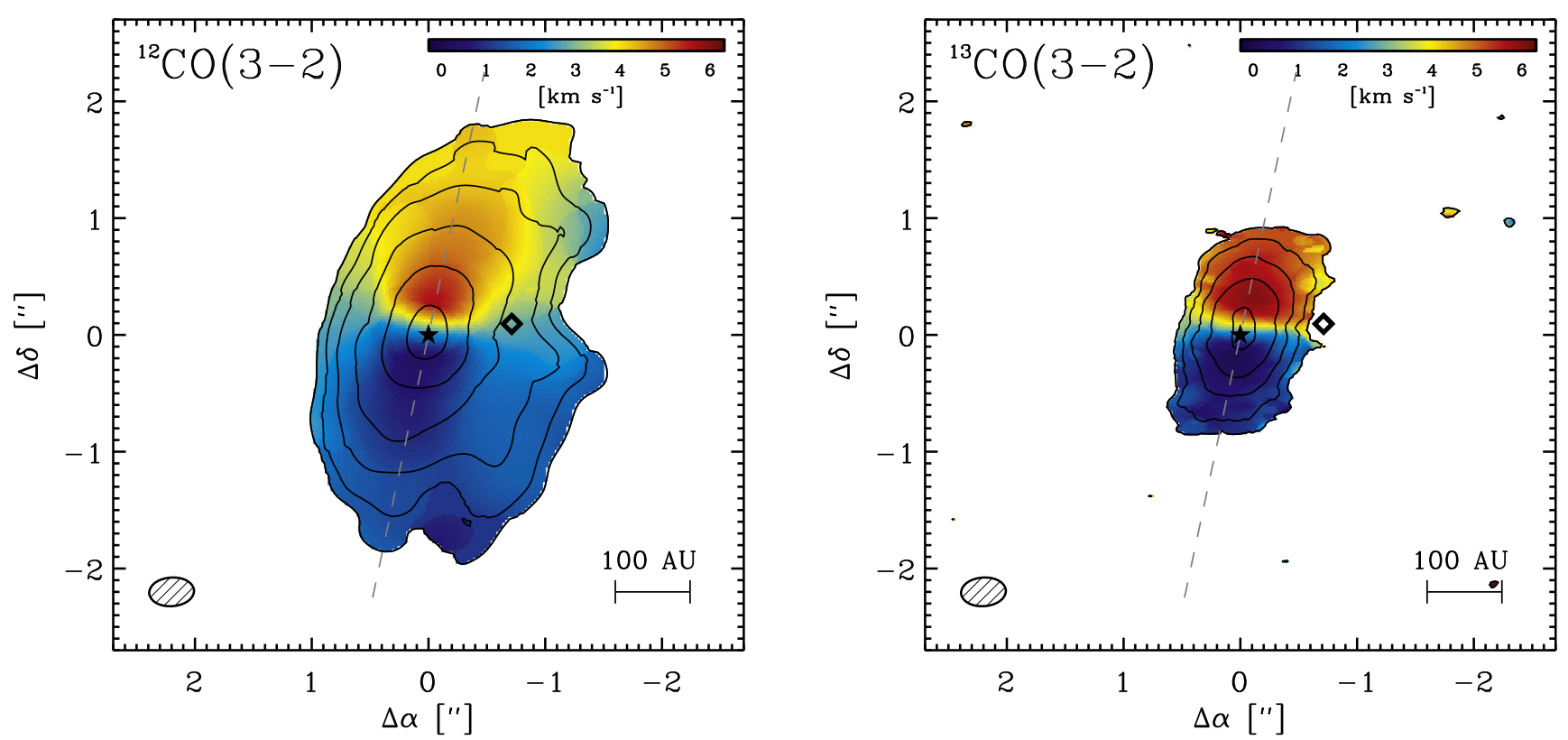

Figure 2. (Left) ${ }^{12} \mathrm{CO} J=3-2$ moment maps for the GQ Lup A circumstellar disk. The zeroth moment (velocity-integrated intensity) map is indicated by contours in steps of $[3,6,12,24,48, \ldots] \times 11 \mathrm{mJy} \mathrm{km} \mathrm{s}^{-1}$ beam $^{-1}$, the $\mathrm{rms}$ noise level in the image. The first moment (intensity-weighted velocity) is shown in color with a scale bar for reference. (Right) The ${ }^{13} \mathrm{CO} J=3-2$ moment maps for the GQ Lup disk. The zeroth moment map is overlaid with contours in steps of [3, 6, 12, 24, 48, $\ldots] \times 6.5 \mathrm{mJy} \mathrm{km} \mathrm{s}^{-1}$ beam $^{-1}$, the rms noise level in the image. Again, the first moment is shown in color with a scale bar for reference. In both panels, the star symbol marks the position of the primary star and the diamond indicates the projected location of the secondary companion. The dashed gray line shows the position angle, $\mathrm{PA}=346^{\circ}$, of the disk major axis determined from modeling and the dashed ellipse indicates the 0 !. $37 \times 00^{\prime \prime} 23$ (FWHM) synthesized beam size.

during the final stages of growth of the host planet, so we cannot rule out the future formation of rocky moons.

If we assume optically thick dust emission for the circumplanetary disk, then we can derive an upper limit on its size. In this limit, the intensity, $I_{\nu}$, is approximately $B_{\nu}\left(T_{\text {dust }}\right)$. Thus,

$$
R_{\text {dust }}=\sqrt{\frac{F_{\nu} D^{2}}{\pi B_{\nu}\left(T_{\text {dust }}\right)}}
$$

Given the upper limit of $F_{\nu}<0.15 \mathrm{mJy}$ and a dust temperature of $18 \mathrm{~K}, R_{\text {dust }}$ must be $<1.1$ au. For comparison, the Hill radius of GQ Lup b assuming a semimajor axis of $\sim 100$ au and an eccentricity of $\sim 0.2$ is $R_{\mathrm{Hill}} \sim 12-19$ au (for companion masses between 10 and $40 M_{\text {Jup }}$ ). Thus, this small disk size may be compatible with numerical simulations of circumplanetary accretion disks that are thick, dense, and truncated at a few tenths of the Hill radius $\left(R \sim 0.3-0.4 R_{\text {Hill }}\right)$ by the gravity of the central star (e.g., Ayliffe \& Bate 2009; Martin \& Lubow 2011; Szulágyi et al. 2016).

\section{2. ${ }^{12} \mathrm{CO}$ and ${ }^{13} \mathrm{CO}$ Emission}

Figure 2 shows the velocity-integrated intensity (0th moment) overlaid as contours on the intensity-weighted velocity (1st moment) for both the ${ }^{12} \mathrm{CO}$ and ${ }^{13} \mathrm{CO}$ emission (left and right panels, respectively). Both maps show a clear pattern of Keplerian rotation, seen more explicitly in the channel maps shown in Figure 3 (top: ${ }^{12} \mathrm{CO}$, bottom: ${ }^{13} \mathrm{CO}$ ). Only the central 11 channels are shown for each line, where emission is clearly resolved at $>3 \sigma$. For the ${ }^{12} \mathrm{CO}$ image, the typical rms in a given channel is $11 \mathrm{mJy} /$ beam (higher due to calibration issues in the spectral window containing ${ }^{12} \mathrm{CO}$ for two of the scheduling blocks that were dealt with by ALMA staff). The integrated and peak intensity are $14.5 \mathrm{Jy} \mathrm{km} \mathrm{s}^{-1}$ and
$1.43 \mathrm{Jy} /$ beam $(130 \sigma)$, respectively. For the ${ }^{13} \mathrm{CO}$ image, the typical rms noise is $6.5 \mathrm{mJy} / \mathrm{beam}$. The integrated and peak intensity are $1.76 \mathrm{Jy} \mathrm{km} \mathrm{s}^{-1}$ and $0.35 \mathrm{Jy} /$ beam $(54 \sigma)$, respectively. The systemic velocity in the LSRK frame is $3.00 \pm 0.01 \mathrm{~km} \mathrm{~s}^{-1}$, and corresponds to $-2.88 \pm 0.01 \mathrm{~km} \mathrm{~s}^{-1}$ in the barycentric frame. Schwarz et al. (2016) recently derived a comparable systemic velocity for the primary of -2.8 $\pm 0.2 \mathrm{~km} \mathrm{~s}^{-1}$ from near-infrared observations using the CRIRES instrument on the VLT.

The CO emission morphology does not show any indication of truncation of the circumprimary gas disk due to the companion, GQ Lup b. Both the ${ }^{12} \mathrm{CO}$ and ${ }^{13} \mathrm{CO}$ emission appear largely symmetric in their spatial distribution across the disk major axis (position angle $\sim 348^{\circ}$ ). There is an indentation and compact $>6 \sigma$ emission peak visible northwest of the star in the ${ }^{12} \mathrm{CO}$ moment and channel maps with velocities between 2 and $3 \mathrm{~km} \mathrm{~s}^{-1}$. Extended interstellar molecular cloud material was seen by van Kempen et al. (2007) in single dish ${ }^{12} \mathrm{CO}$ emission towards GQ Lup with $v_{\mathrm{LSRK}} \sim 4-5 \mathrm{~km} \mathrm{~s}^{-1}$. Although the velocities of the observed structure and the extended interstellar component do not match exactly, it is plausible that the ${ }^{12} \mathrm{CO}$ ALMA images of the circumprimary disk are affected by contamination from ambient cloud emission.

In order to determine a dynamical mass for GQ Lup A and to characterize the gas disk geometrical properties, we forwardmodel the ${ }^{12} \mathrm{CO}$ and ${ }^{13} \mathrm{CO}$ molecular line emission using the DiskJockey package ${ }^{8}$ (Czekala et al. 2015). We adopt a simple parametric model of disk structure, which uses a selfsimilar surface density profile (Lynden-Bell \& Pringle 1974) described by a characteristic radius, $r_{c}$, and total gas mass,

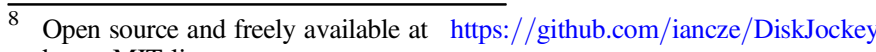
under an MIT license.
} 

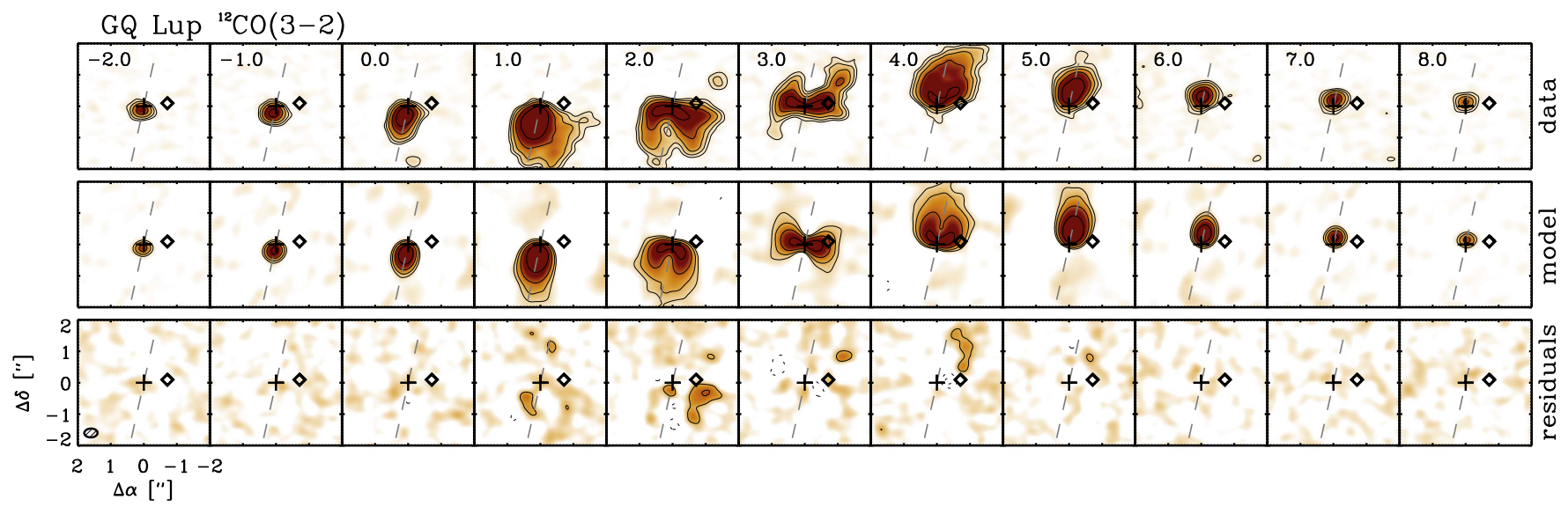

$\Delta \alpha["]$

GQ Lup ${ }^{13} \mathrm{CO}(3-2)$
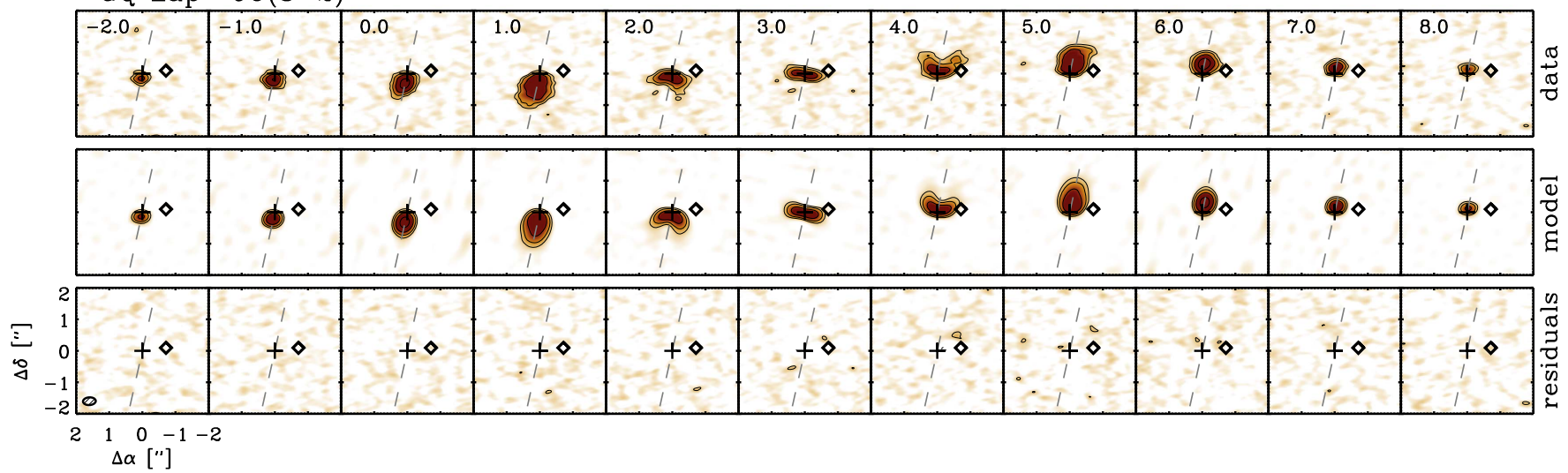

Figure 3. Channel maps (top row), the best-fit model (model row), and the imaged residuals (bottom row) for both the ${ }^{12} \mathrm{CO}$ (top) and ${ }^{13} \mathrm{CO}$ (bottom) $J=3-2$ emission for GQ Lup. Contours for all panels are in steps of $[3,6,12,24,48, \ldots] \times$ the rms noise level in the image, with an $\mathrm{rms}$ of $11 \mathrm{mJy} / \mathrm{beam}$ and $6.5 \mathrm{mJy} /$ beam for the ${ }^{12} \mathrm{CO}$ and ${ }^{13} \mathrm{CO}$ images, respectively. The ellipse in the lower left corner of both bottom leftmost panels indicates the $00^{\prime \prime} 37 \times 0$.! 23 (FWHM) synthesized beam size. Each channel is $1 \mathrm{~km} \mathrm{~s}^{-1}$ wide with the LSR velocities labeled in the upper left corner of each panel.

$M_{\text {gas: }}$

$$
\Sigma=\Sigma_{c}\left(\frac{r}{r_{c}}\right)^{-1} \exp \left[-\left(\frac{r}{r_{c}}\right)^{2}\right]
$$

Here, $\Sigma_{c}$ is a normalization given by $e \times \Sigma\left(r_{c}\right), M_{\text {gas }}=$ $X_{\mathrm{CO}} \Sigma_{c}\left(2 \pi r_{c}^{2}\right)$, and $X_{\mathrm{CO}}$ is the fractional abundance of $\mathrm{CO}$ (assumed to be constant throughout the disk). The disk is assumed to be vertically isothermal and in hydrostatic equilibrium, with a radial power-law index, $q$, and a normalization at $10 \mathrm{au}, T_{10}$ :

$$
T=T_{10}\left(\frac{r}{10 \mathrm{au}}\right)^{-q}
$$

The velocity field is assumed to be Keplerian with systemic velocity, $v_{\text {sys }}$, and is dominated by the stellar mass, $M_{*}$. Nonthermal (turbulent) line broadening is denoted by a constant velocity width, $\xi$. We also include two offsets in both R.A. and decl., $\Delta \alpha$ and $\Delta \delta$, respectively. The posterior probability of the model parameters is evaluated in the following manner: (1) sky images of a given disk structure are generated using the RADMC $-3 \mathrm{D}$ radiative transfer program (Dullemond 2012), Fourier transformed, and sampled at the $u$, $v$ locations corresponding to the ALMA baselines, and (2) the model visibilities are then evaluated using a $\chi^{2}$ likelihood function which incorporates the statistical weights on each visibility measurement. This generative model allows us to fully explore the uncertainties in each parameter as well as determine the one-dimensional marginalized probability distribution on stellar mass. Although more sophisticated models of disk structure are desirable, this simple model has been proven to yield accurate stellar masses, as confirmed by comparison with measurements of circumbinary disks around spectroscopic binaries (Rosenfeld et al. 2012; Czekala et al. 2015, 2016). Further details of the modeling framework can be found in Czekala et al. (2015).

The best-fit parameter values and their $68 \%$ uncertainties are listed in Table 2. Figure 3 shows the channel maps for the data (top), best-fit model imaged like the data (middle), and resulting residuals (bottom) for both the ${ }^{12} \mathrm{CO}$ (top grouping) and ${ }^{13} \mathrm{CO}$ (bottom grouping) emission. The results for both lines are consistent, although the ${ }^{12} \mathrm{CO}$ fits may be biased by the cloud contamination evident in the residuals (see channels with velocities between 1 and $4 \mathrm{~km} \mathrm{~s}^{-1}$ ). As a result, we focus on the best-fit parameters from the ${ }^{13} \mathrm{CO}$ modeling.

The models imply a stellar mass, $M_{*}$, for GQ Lup A of $1.03 \pm 0.15 M_{\odot}$. The quoted uncertainty on the mass includes the significant uncertainty in the distance $( \pm 50 \mathrm{pc})$ added in quadrature. At a known distance, $d$, the constraint on the stellar mass can be recast as $M_{*}=(1.03 \pm 0.05) *(d / 156 \mathrm{pc})$, where the formal uncertainty on $M_{*}$ is $\sim 5 \%$ including systematic uncertainties estimated from more complex models (e.g., vertical structure, see Rosenfeld et al. 2013). Previous 
Table 2

${ }^{12} \mathrm{CO}$ and ${ }^{13} \mathrm{CO}$ Model Parameters

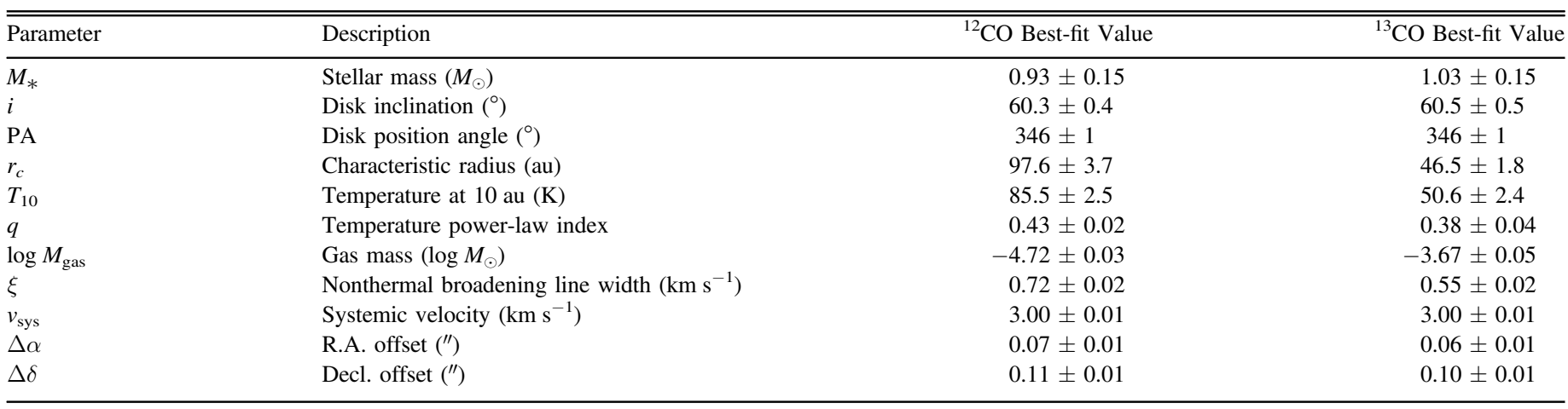

estimates of the stellar mass of GQ Lup A from the literature are mostly lower than our determination, ranging between 0.7 and $1.05 M_{\odot}$ (see discussion in Section 2, Mugrauer \& Neuhäuser 2005; Seperuelo Duarte et al. 2008; Donati et al. 2012). The discrepancy in mass estimates results largely from differences in stellar evolutionary models and uncertainty in the effective temperature. Given this result and previous work (Rosenfeld et al. 2013; Czekala et al. 2015, 2016), ALMA can play a substantial role in precisely measuring the masses of large samples of young stars, providing constraints on evolutionary models. Much work has been done to determine allowable orbits for the companion, GQ Lup b, all of which assume a stellar mass of $0.7 M_{\odot}$ (Ginski et al. 2014; Pearce et al. 2015; Schwarz et al. 2016). Pearce et al. (2015) define a criteria for a bound orbit, $B<1$, where $B \propto$ $\left(M / M_{\odot}\right)^{-1}$. This new determination of the stellar mass of GQ Lup A may prove relevant for constraining allowable orbits of the secondary.

The characteristic radius and total gas mass for the best-fit model to the ${ }^{13} \mathrm{CO}$ emission are $46.5 \pm 1.8 \mathrm{au}$ and $\log M_{\text {gas }} / M_{\odot}=-3.67 \pm 0.05$, respectively. We also compare our ${ }^{12} \mathrm{CO}$ and ${ }^{13} \mathrm{CO}$ integrated line intensities to the model grids of Williams \& Best (2014), which predict a gas mass between $10^{-4}$ and $10^{-3} M_{\odot}$, consistent with our modeling results. By combining this gas mass with the total dust mass determination from Section 4.1, we can calculate the gas-todust ratio for the circumstellar disk around GQ Lup A to be $4.7 \pm 0.5$. This result is well below ISM gas-to-dust ratios, but is comparable to measurements made by Ansdell et al. (2016) for circumstellar disks around other T Tauri stars in Lupus with similar stellar masses. In fact, nearly all of the detected Lupus disks are inferred to have gas-to-dust ratios well below 100. A significant caveat to our derived gas mass is that it depends inversely on the $\mathrm{CO} / \mathrm{H}_{2}$ abundance ratio, which we assume to be ISM-like $\sim 10^{-4}$. Furthermore, recent work by Miotello et al. (2016) suggests that a more complex analysis is required to accurately determine disk gas masses.

\section{DISCUSSION}

We have performed interferometric observations of the GQ Lup system at $870 \mu \mathrm{m}$ with ALMA and detected both continuum and ${ }^{12} \mathrm{CO}$ and ${ }^{13} \mathrm{CO} J=3-2$ line emission. The continuum image reveals compact dust emission surrounding the primary star, but no emission at the position of the secondary companion. We place a robust $3 \sigma$ upper limit on the flux of a circumplanetary disk surrounding the companion of $<0.15 \mathrm{mJy}$. We use the Keplerian velocity field as determined by the line emission data to estimate the mass of the primary star, $M_{*}=1.03 \pm 0.15 M_{\odot}$, and the geometry of the circumprimary disk. We now use this new information to discuss the geometry of the circumprimary disk and implications for formation scenarios of massive companions on wide orbits.

\subsection{Circumprimary Disk Geometry}

The CO emission models place tight constraints on the geometry of the circumprimary disk through the inclination and position angle. For the ${ }^{13} \mathrm{CO}$ emission, the best-fit inclination is $i=60^{\circ} .5 \pm 0.5$ and position angle is $\mathrm{PA}=346^{\circ} \pm 1^{\circ}$. There are discrepancies in the literature over the inclination angles of the stellar rotation axis and circumprimary disk for the GQ Lup system. Broeg et al. (2007) combine photometric rotation period monitoring with a previous measurement of $v \sin i$ from HARPS (Guenther et al. 2005) to determine the inclination of the star's rotational axis to be $i=27^{\circ} \pm 5^{\circ}$, much lower than the inclination of the circumstellar disk. In contrast, Seperuelo Duarte et al. (2008) derive a higher inclination of $53^{\circ} \pm 18^{\circ}$ from spectrophotometric data taken with the $1.52 \mathrm{~m} \mathrm{ESO}$ telescope in La Silla. Using high-resolution VLT/CRIRES spectra of $\mathrm{CO}$ emission from GQ Lup, Pontoppidan et al. (2011) find a best-fit disk inclination of $65^{\circ} \pm 10^{\circ}$. Our analysis agrees with these later determinations of the disk inclination and suggests that the disk inclination is significantly higher than previously estimated for the star.

Assuming that the orbit of GQ Lup b is also coplanar with the circumprimary disk implies that the current physical separation of GQ Lup b could be as large as $~ 220$ au. Schwarz et al. (2016) and Ginski et al. (2014) (assuming a stellar mass of $0.7 M_{\odot}$ ) propose three families of orbital solutions for GQ Lup b: (1) semimajor axis $\sim 100 \mathrm{au}, i \sim 57^{\circ}$, eccentricity $\sim 0.15$, (2) semimajor axis $<185 \mathrm{au}, 28^{\circ}<i<63^{\circ}$, eccentricity 0.2 to 0.75 , and (3) semimajor axis $>300 \mathrm{au}$, $52^{\circ}<i<63^{\circ}$, eccentricity $>0.8$. More specifically, they note that orbits with lower eccentricities between 0.1 and 0.4 have high inclinations between $48^{\circ}$ and $63^{\circ}$. Given the apparent discrepancy between these high inclinations and the assumed low inclination of the circumstellar disk $\left(i \sim 27^{\circ}\right)$, Schwarz et al. (2016) and Ginski et al. (2014) suggest that GQ Lup b was likely scattered to its current position since in situ formation would result in a low eccentricity orbit near the plane of the circumstellar disk. Our new robust measurement of the circumstellar disk inclination relieves some of this tension and does not exclude an in situ formation, since an inclination of $60^{\circ} .5 \pm 0.5$ is well within the range determined for low eccentricity orbits. 


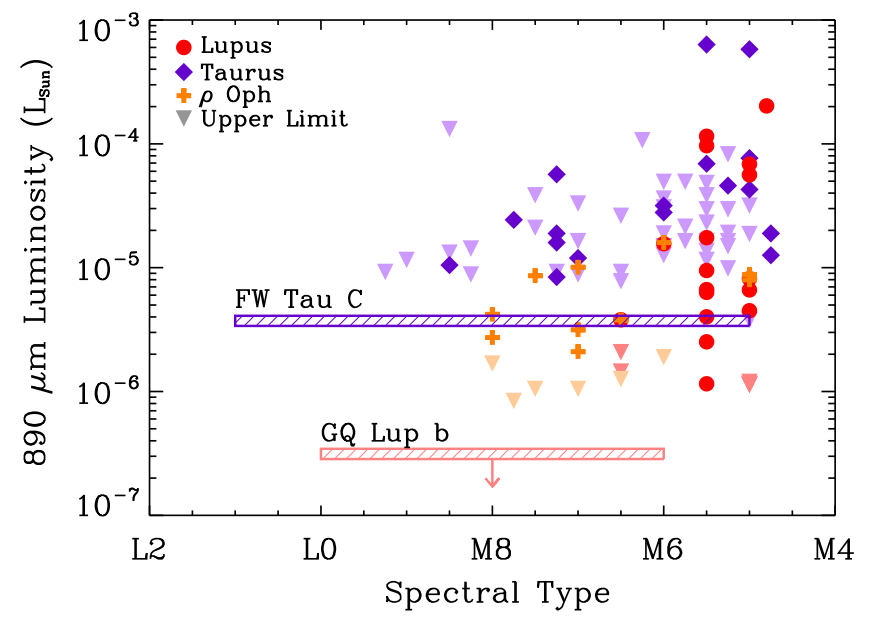

Figure 4. (Sub)millimeter dust luminosities as a function of spectral type for the young ( $\sim 2$ Myr old) Lupus (red circles, Ansdell et al. 2016), Taurus (purple diamonds, Andrews et al. 2013; Ricci et al. 2014), and $\rho$ Ophiucus (orange crosses, Testi et al. 2016) star-forming regions. The upside-down triangles indicate $3 \sigma$ upper limits. Our upper limit for GQ Lup b is nearly an order of magnitude lower than the previous ALMA measurement of a circumplanetary disk surrounding FW Tau C (Kraus et al. 2015).

\subsection{Comparison to Other Young Substellar Objects}

The $3 \sigma$ dust mass upper limit we obtain for GQ Lup b is lower than previous circumplanetary disk mass constraints obtained with ALMA. Bowler et al. (2015) observed GSC 6214-210, a 5-10 Myr old system with a $\sim 15 M_{\text {Jup }}$ companion at a separation of $\sim 320$ au and did not detect dust emission surrounding either the primary or secondary; they place an upper limit on the circumplanetary dust mass of $<0.15 M_{\oplus}$ or $<0.3 \%$ of the companion mass. However, a non-detection of millimeter dust emission around both the primary and the secondary is consistent with the results of a large survey of the TW Hya association, which found dust masses for similar late spectral-type objects of $\lesssim 10^{-2} M_{\oplus}$ (Rodriguez et al. 2015). In older systems like these, it is possible that the effects of grain growth and drift have depleted the disks of grains that are emissive at millimeter wavelengths. In contrast, ALMA observations of the younger, 2 Myr old FW Tau system (Kraus et al. 2015) detected significant dust emission surrounding the $<40 M_{\text {Jup }}$ companion at $\sim 330$ au (Caceres et al. 2015), implying a circumplanetary dust mass of $1-2 M_{\oplus}$. However, the spectral energy distribution, especially at near-infrared wavelengths, suggests that FW Tau $\mathrm{C}$ is degenerate between a planetary mass object and a very low-mass star or brown dwarf (spectral type M5-M8) with an edge-on disk (Bowler et al. 2014). Figure 4 compares our ALMA constraint on the dust luminosity of a circumplanetary disk around GQ Lup b to the previous constraints on FW Tau C from Kraus et al. (2015). Also included in Figure 4 are previous (sub)millimeter measurements for sources with spectral types M5 and later from surveys of the young ( $\sim 2$ Myr old) Lupus, Taurus, and $\rho$ Ophiucus star-forming regions (Andrews et al. 2013; Ricci et al. 2014; Ansdell et al. 2016; Testi et al. 2016). All of the dust luminosities were calculated from measurements of the $890 \mu \mathrm{m}$ flux density. To construct this plot, we assumed the mean distance for each star-forming region to be the following: $156 \pm 50$ pc (Lupus I, II, IV, Neuhäuser et al. 2008), $200 \pm 50$ pc (Lupus III Comerón 2008), $140 \pm 20$ pc (Taurus, Torres et al. 2012), and $135 \pm 8$ pc ( $\rho$ Ophiucus, Mamajek 2008). The ALMA limit for GQ Lup b is nearly an order of magnitude lower than the detections from these other large surveys. This wide spread in dust luminosity for similar spectral-type objects shows that there is a wide range of evolutionary outcomes for circumstellar disks at these young ages.

\subsection{Implications for Formation Scenarios}

The growing sample of systems with deep millimeter observations and corresponding limits on circumplanetary disk masses allows us to speculate on proposed formation mechanisms of such systems (Debes \& Sigurdsson 2006; Dai et al. 2010). One possibility is that these wide-separation substellar companions formed in situ through core fragmentation or gravitational instability. However, models predict that companions formed through these mechanisms should be surrounded by massive circumplanetary disks that persist over several Myr by accreting material from the disk of the parent star (Boley 2009; Vorobyov \& Basu 2010; Stamatellos \& Herczeg 2015). Another possibility is that these substellar companions formed much closer in to the primary star and were later scattered outward through dynamical interactions with another massive body (Boss 2006; Crida et al. 2009; Scharf \& Menou 2009). Such chaotic events are likely to disrupt or destroy any circumplanetary disk surrounding the companion, since the closest approach is $\ll R_{\text {Hill. }}$. However, a recent survey by Bryan et al. (2016) of eight wide-separation planetary mass companions ruled out the presence of $<7 \quad M_{\text {Jup }}$ inner companions in these systems at separations of 15-50 au, suggesting that scattering may not be a dominant mechanism for the formation of wide-separation companions. It is also possible that such systems formed through the standard binary fragmentation route (Fisher 2004; Offner et al. 2010; Bate 2012), where turbulent fragmentation and orbit evolution can result in wide-separation, unequal-mass binary systems.

While the null detection of a circumplanetary disk around GQ Lup b $\left(M_{\text {dust }}<0.04 M_{\oplus}\right)$ argues against in situ formation, its orbital parameters are still consistent with such a model. Indeed, the models of Ginski et al. (2014) and Schwarz et al. (2016) do not exclude low eccentricity orbits as would be expected for a planet-like formation within a larger protoplanetary disk. The morphology of the GQ Lup A disk points against a scattering origin for the companion. There is no observational evidence for a sharp inner edge or cavity indicative of an additional companion in the system that may have scattered GQ Lup b out to its current position. Observations with higher angular resolution are needed to probe for any substructure in the circumprimary disk that would signify the influence of an additional companion, or features that may have resulted from a previous scattering event.

Schwarz et al. (2016) measure the projected rotational velocity of GQ Lup b to be $5.3_{-1.0}^{+0.9} \mathrm{~km} \mathrm{~s}^{-1}$, making it a slow rotator compared to the giant planets in the solar system and the recent measurement of $\beta$ Pictoris b (Snellen et al. 2014). Objects formed through gravitational instability or core fragmentation seem to follow a spin-mass trend, where higher mass objects rotate faster than lower mass objects. The unusually slow spin of GQ Lup b could point to a different formation scenario, but, as Schwarz et al. (2016) point out, GQ Lup b is still quite young and will likely spin up over time, making its slow spin less discrepant. 


\section{CONCLUSIONS}

We present new ALMA observations of $870 \mu \mathrm{m}$ dust continuum and CO $J=3-2$ line emission from the GQ Lup system. These observations resolve the circumstellar disk surrounding GQ Lup A, and provide a deep upper limit on any emission from a circumplanetary disk surrounding GQ Lup b. The main results are as follows.

1. The circumprimary disk appears compact with a FWHM of $59 \pm 12$ au. Given the total flux density and assuming optically thin emission, we determine a total dust mass of $M_{\text {dust }}=15.10 \pm 0.04 M_{\oplus}$.

2. There is no indication that the circumprimary disk traced by ${ }^{12} \mathrm{CO}$ and ${ }^{13} \mathrm{CO}$ emission is truncated or affected by the presence of the companion, GQ Lup b. The characteristic radius of the ${ }^{13} \mathrm{CO}$ emission is $46.5 \pm 1.8 \mathrm{au}$, more extended than the dust disk. By forward-modeling the Keplerian velocity field, we robustly constrain both the mass of the primary star, $M_{*}=(1.03 \pm 0.05) *(d / 156 \mathrm{pc})$, and the geometry of the circumprimary disk, $i=$ $60.5 \pm 0.5$ and $\mathrm{PA}=346^{\circ} \pm 1^{\circ}$. An inclination of $i=60^{\circ}$ is significantly higher than previous estimates of $20^{\circ}-30^{\circ}$. If the companion orbit is coplanar with the circumprimary disk, then this high inclination implies that the current physical separation of the secondary is $\sim 220$ au.

3. We determine a robust $3 \sigma$ upper limit on the flux density of any circumplanetary disk surrounding GQ Lup b of $<0.15 \mathrm{mJy}$. If we assume optically thin emission, then this implies an upper limit on the dust mass of $M_{\text {dust }}<0.04 M_{\oplus}$. This limit is an order of magnitude lower than previous ALMA measurements for circumstellar disks around M5 and later sources of similar ages ( $\sim 2 \mathrm{Myr}$ ). In the optically thick limit, we can instead derive an upper limit on the radius of the circumplanetary disk of $R_{\text {dust }}<1.1$ au.

4. Since models of in situ formation of wide-separation, substellar companions through core fragmentation or gravitational instability predict massive circumplanetary disks that persist for several Myr, the lack of detections of such massive disks disfavors these formation scenarios.

Millimeter observations of additional systems with young substellar companions are needed to characterize the disk properties and to assess whether or not the features of the GQ Lup system are typical of the whole population. In addition, higher angular resolution is needed to probe for any substructure in circumprimary disks, like GQ Lup, that could indicate the presence of additional companions involved in dynamical evolution.

M.A.M. acknowledges support from a National Science Foundation Graduate Research Fellowship (DGE1144152) and from NRAO Student Observing Support. This paper makes use of the following ALMA data: ADS/JAO.ALMA \#2013.1.00374.S. ALMA is a partnership of ESO (representing its member states), NSF (USA), and NINS (Japan), together with NRC (Canada) and NSC and ASIAA (Taiwan) and KASI (Republic of Korea), in cooperation with the Republic of Chile. The Joint ALMA Observatory is operated by ESO, AUI/ NRAO and NAOJ. The National Radio Astronomy Observatory is a facility of the National Science Foundation operated under cooperative agreement by Associated Universities, Inc.
This work has also made use of data from the European Space Agency (ESA) mission Gaia (http://www.cosmos.esa.int/ gaia), processed by the Gaia Data Processing and Analysis Consortium (DPAC, http://www.cosmos.esa.int/web/gaia/ dpac/consortium). Funding for the DPAC has been provided by national institutions, in particular the institutions participating in the Gaia Multilateral Agreement.

\section{REFERENCES}

Alcalá, J. M., Natta, A., Manara, C. F., et al. 2014, A\&A, 561, A2

Andrews, S. M., Rosenfeld, K. A., Kraus, A. L., \& Wilner, D. J. 2013, ApJ, 771,129

Ansdell, M., Williams, J. P., van der Marel, N., et al. 2016, arXiv:1604.05719

Ayliffe, B. A., \& Bate, M. R. 2009, MNRAS, 397, 657

Bailey, V., Hinz, P. M., Currie, T., et al. 2013, ApJ, 767, 31

Bate, M. R. 2012, MNRAS, 419, 3115

Bate, M. R., Bonnell, I. A., \& Bromm, V. 2003, MNRAS, 339, 577

Beckwith, S. V. W., Sargent, A. I., Chini, R. S., \& Guesten, R. 1990, AJ, 99, 924

Boley, A. C. 2009, ApJL, 695, L53

Boley, A. C., Hayfield, T., Mayer, L., \& Durisen, R. H. 2010, Icar, 207, 509 Boss, A. P. 2006, ApJL, 637, L137

Bowler, B. P., Andrews, S. M., Kraus, A. L., et al. 2015, ApJL, 805, L17

Bowler, B. P., Liu, M. C., Kraus, A. L., \& Mann, A. W. 2014, ApJ, 784, 65

Bowler, B. P., Liu, M. C., Kraus, A. L., Mann, A. W., \& Ireland, M. J. 2011, ApJ, 743, 148

Broeg, C., Schmidt, T. O. B., Guenther, E., et al. 2007, A\&A, 468, 1039

Bryan, M. L., Bowler, B. P., Knutson, H. A., et al. 2016, ApJ, 827, 100

Caceres, C., Hardy, A., Schreiber, M. R., et al. 2015, ApJL, 806, L22

Canup, R. M., \& Ward, W. R. 2006, Natur, 441, 834

Chauvin, G., Lagrange, A.-M., Dumas, C., et al. 2005, A\&A, 438, L25

Comerón, F. 2008, in Handbook of Star Forming Regions, Volume II: The Southern Sky ASP Monograph Publications, The Lupus Clouds, ed. B. Reipurth (San Francisco, CA: ASP), 295

Crida, A., Masset, F., \& Morbidelli, A. 2009, ApJL, 705, L148

Czekala, I., Andrews, S. M., Jensen, E. L. N., et al. 2015, ApJ, 806, 154

Czekala, I., Andrews, S. M., Torres, G., et al. 2016, ApJ, 818, 156

Dai, Y., Wilner, D. J., Andrews, S. M., \& Ohashi, N. 2010, AJ, 139, 626

Debes, J. H., \& Sigurdsson, S. 2006, A\&A, 451, 351

Donati, J.-F., Gregory, S. G., Alencar, S. H. P., et al. 2012, MNRAS, 425, 2948

Dullemond, C. P. 2012, RADMC-3D: A Multi-Purpose Radiative Transfer Tool, Astrophysics Source Code Library, ascl:1202.015

Fisher, R. T. 2004, ApJ, 600, 769

Ginski, C., Schmidt, T. O. B., Mugrauer, M., et al. 2014, MNRAS, 444, 2280

Guenther, E. W., Neuhäuser, R., Wuchterl, G., et al. 2005, AN, 326, 958

Heller, R., Williams, D., Kipping, D., et al. 2014, AsBio, 14, 798

Herczeg, G. J., \& Hillenbrand, L. A. 2014, ApJ, 786, 97

Hildebrand, R. H. 1983, QJRAS, 24, 267

Hogerheijde, M. R., Bekkers, D., Pinilla, P., et al. 2016, A\&A, 586, A99

Hughes, J., Hartigan, P., Krautter, J., \& Kelemen, J. 1994, AJ, 108, 1071

Ireland, M. J., Kraus, A., Martinache, F., Law, N., \& Hillenbrand, L. A. 2011, ApJ, 726, 113

Isella, A., Chandler, C. J., Carpenter, J. M., Pérez, L. M., \& Ricci, L. 2014, ApJ, 788, 129

Jiang, I.-G., Laughlin, G., \& Lin, D. N. C. 2004, AJ, 127, 455

Kharchenko, N. V., \& Roeser, S. 2009, VizieR Online Data Catalog: All-Sky Compiled Catalogue of 2.5 million stars

Kratter, K. M., Murray-Clay, R. A., \& Youdin, A. N. 2010, ApJ, 710, 1375

Kraus, A. L., Andrews, S. M., Bowler, B. P., et al. 2015, ApJL, 798, L23

Kraus, A. L., Ireland, M. J., Cieza, L. A., et al. 2014, ApJ, 781, 20

Lafrenière, D., Jayawardhana, R., \& van Kerkwijk, M. H. 2008, ApJL, 689, L153

Lambrechts, M., \& Johansen, A. 2012, A\&A, 544, A32

Lavigne, J.-F., Doyon, R., Lafrenière, D., Marois, C., \& Barman, T. 2009, ApJ, 704, 1098

Lindegren, L., Lammers, U., Bastian, U., et al. 2016, arXiv:1609.04303

Luhman, K. L., Wilson, J. C., Brandner, W., et al. 2006, ApJ, 649, 894

Lynden-Bell, D., \& Pringle, J. E. 1974, MNRAS, 168, 603

Mamajek, E. E. 2008, AN, 329, 10

Mamajek, E. E., Quillen, A. C., Pecaut, M. J., et al. 2012, AJ, 143, 72

Marois, C., Macintosh, B., \& Barman, T. 2007, ApJL, 654, L151 
Martin, R. G., \& Lubow, S. H. 2011, MNRAS, 413, 1447

Miotello, A., van Dishoeck, E. F., Kama, M., \& Bruderer, S. 2016, arXiv:1605. 07780

Mugrauer, M., \& Neuhäuser, R. 2005, AN, 326, 701

Neuhäuser, R., Guenther, E. W., Wuchterl, G., et al. 2005, A\&A, 435, L13

Neuhäuser, R., Mugrauer, M., Seifahrt, A., Schmidt, T. O. B., \& Vogt, N. 2008, A\&A, 484, 281

Nuernberger, D., Chini, R., \& Zinnecker, H. 1997, A\&A, 324, 1036

Offner, S. S. R., Kratter, K. M., Matzner, C. D., Krumholz, M. R., \& Klein, R. I. 2010, ApJ, 725, 1485

Pearce, T. D., Wyatt, M. C., \& Kennedy, G. M. 2015, MNRAS, 448, 3679

Pecaut, M. J., \& Mamajek, E. E. 2013, ApJS, 208, 9

Pollack, J. B., Hubickyj, O., Bodenheimer, P., et al. 1996, Icar, 124, 62

Pontoppidan, K. M., Blake, G. A., \& Smette, A. 2011, ApJ, 733, 84

Ricci, L., Testi, L., Natta, A., et al. 2014, ApJ, 791, 20

Rodriguez, D. R., van der Plas, G., Kastner, J. H., et al. 2015, A\&A, 582, L5

Rosenfeld, K. A., Andrews, S. M., Hughes, A. M., Wilner, D. J., \& Qi, C. 2013, ApJ, 774, 16

Rosenfeld, K. A., Andrews, S. M., Wilner, D. J., \& Stempels, H. C. 2012, ApJ, 759,119
Scharf, C., \& Menou, K. 2009, ApJL, 693, L113

Schmidt, T. O. B., Neuhäuser, R., Seifahrt, A., et al. 2008, A\&A, 491, 311

Schwarz, H., Ginski, C., de Kok, R. J., et al. 2016, arXiv:1607.00012

Seifahrt, A., Neuhäuser, R., \& Hauschildt, P. H. 2007, A\&A, 463, 309

Seperuelo Duarte, E., Alencar, S. H. P., Batalha, C., \& Lopes, D. 2008, A\&A, 489,349

Snellen, I. A. G., Brandl, B. R., de Kok, R. J., et al. 2014, Natur, 509, 63

Stamatellos, D., \& Herczeg, G. J. 2015, MNRAS, 449, 3432

Szulágyi, J., Masset, F., Lega, E., et al. 2016, MNRAS, 460, 2853

Tachihara, K., Dobashi, K., Mizuno, A., Ogawa, H., \& Fukui, Y. 1996, PASJ, 48, 489

Testi, L., Natta, A., Scholz, A., et al. 2016, arXiv:1606.06448

Torres, R. M., Loinard, L., Mioduszewski, A. J., et al. 2012, ApJ, 747, 18

van der Plas, G., Ménard, F., Ward-Duong, K., et al. 2016, ApJ, 819, 102

van Kempen, T. A., van Dishoeck, E. F., Brinch, C., \& Hogerheijde, M. R. 2007, A\&A, 461, 983

Vorobyov, E. I., \& Basu, S. 2010, ApJL, 714, L133

Williams, J. P., \& Best, W. M. J. 2014, ApJ, 788, 59

Zhou, Y., Herczeg, G. J., Kraus, A. L., Metchev, S., \& Cruz, K. L. 2014, ApJL, 783, L17 